\title{
Estudio cualitativo a pacientes de cáncer con soporte paliativo en atención hospitalaria
}

\author{
A qualitative study of cancer patients \\ receiving palliative support in hospital care
}

${ }^{1}$ Escuela Universitaria de Enfermería, Universidad de Barcelona (EUE.UB). Campus de Bellvitge Pavelló de Govern, $3^{\text {a }} \mathrm{pl}$. 08907 L'Hospitalet de Llobregat. Barcelona España.mgetino@ub.edu
Abstract This article focuses on patients with cancer during the terminal phase. It deals with the care that individuals need after trying to seek health solutions in different hospitals when the disease is deemed incurable and they then require palliative care $(P C)$ to obtain welfare and assist them to die. In the Spanish health system these patients are cared for in palliative care units or services. The main objective of palliative care is to give comfort. Comforting is destined to alleviate and support patients and families during the process of health / disease / care / patient death. During this period, it is important to care for the ailing body. Similarly, when a disease is incurable, the pain and suffering runs its inexorable course and leaves perceptible traces. These signs can be an indication that death is nigh. Although scientific advances have produced positive results in the control of pain, in some cases there are critical moments when situations of misery and despair arise for the terminal patients.

Key words Palliative care, Cancer patient, End of life, Body, Pain, Death, Qualitative research
Resumen Este artículo se centra en el enfermo con cáncer en situación terminal. Trata de los cuidados que requiere la persona que tras haber realizado un recorrido hospitalario en busqueda de salud es considerada incurable $y$ dirigida a cuidados paliativos (CP) para procurarle bienestar y ayudarle a morir. En la geografía española estos enfermos son atendidos en unidades o servicios de cuidados paliativos (SCP). El objetivo principal en cuidados paliativos es cuidar. Cuidar es aliviar y acompañar al paciente, y dar soporte a los familiares durante el proceso de salud/enfermedad/atención/ muerte del paciente. En el cuidado es importante tratar el cuerpo enfermo. Por lo que prevalece aquello que acontece al afectado y que queda inscrito en su cuerpo. De la misma manera que en la enfermedad incurable el dolor y el sufrimiento marcan un itinerario y trazan unas huellas perceptibles en el mismo. Estos signos pueden resultar una señal que le alerta de la proximidad a la muerte. Aunque los avances científicos han influido, positivamente, en el control del dolor, en algunos casos existen momentos críticos en que se producen situaciones de descontrol y desesperación de quienes lo padecen.

Palabras clave Cuidados paliativos, Enfermedad de cáncer, Final de vida, Cuerpo, Dolor, Muerte, Investigación cualitativa 


\section{Introducción}

En este artículo se presenta la atención paliativa que requieren los pacientes afectados de cáncer y la relación que establecen con el cuerpo en la etapa final de su vida. Durante el proceso de la enfermedad los mensajes que reciben del cuerpo les presentan unos interrogantes, sobre todo, en situaciones de deterioro evidente y sufrimiento, cerciorándose de que les llega su final.

La paliación es el lugar a donde va dirigido el enfermo moribundo para recibir el cuidado paliativo hasta el final de su vida. La hospitalización en el SCP coincide con los periodos de agravamiento y descontrol de los síntomas de la enfermedad. Se plantea un nuevo cambio de actuación centrado en abordar la enfermedad en su fase final. En esta etapa terminal, se reemplaza la terapia curativa por la paliativa. Por lo que, el servicio es el espacio apropiado para observar los procesos de enfermedad/atención/muerte. Este espacio social se convierte en el eje de atención en donde los enfermos y los familiares, a los que se suman los profesionales del equipo interdisciplinar, conviven con la enfermedad terminal e interaccionan entre sí, en un medio en el que dan significado y sentido a la atención paliativa.

La acción de cuidar es extensible al equipo interdisciplinar de CP, pero aún y así, sigue siendo detentada mayoritariamente por los profesionales enfermeros. En este entorno el paciente y los familiares reciben cuidados y apoyo profesionalizados, durante el proceso de terminalidad/ agonía/muerte del paciente, por parte de los profesionales enfermeros de la unidad de cuidados paliativos (UCP). Así pues, cuidar supone que es tan importante ayudar a morir como evitar la muerte, por lo que los cuidados paliativos no son sólo el último recurso de perdedores biológicos a los que la medicina no puede salvar, sino algo a lo que todas las personas pueden aspirar, aunque aún queda un largo camino por recorrer $^{1}$. Los cuidados paliativos realizados por un equipo interdisciplinar ofrecen un sistema de soporte para ayudar a los pacientes a vivir tan activamente como sea posible hasta la muerte y a los familiares a adaptarse durante la enfermedad del paciente y en el duelo².

El cáncer es la enfermedad que no avisa y entra sin llamar, es vivida como una irrupción violenta y oculta. Para Susan Sontag, "esto cambiará cuando se aclare su etiología y su tratamiento sea tan eficaz como ha llegado a ser el de la tuberculosis"3. La enfermedad de cáncer es percibida socialmente como una enfermedad incurable y si avanza es cuando son conscientes de que algo no va bien, que no se curan y pueden morir. Así, el padecer cáncer está considerada una desgracia, confirmando que la desgracia o el infortunio es una de las respuestas a la situación de enfermedad, más allá de las simples implicaciones físicas; por considerarlas como categorías explicativas que tienen en común una caracterización cultural y social ${ }^{2}$. Para las personas que padecen esta enfermedad cerciorarse de que no se van a curar, es un duro golpe, en el que pasan por momentos perturbadores y en el que crean estrategias compensatorias ante la adversidad.

El concepto de muerte presentado como un acontecimiento que ocurre dentro de un orden social. "Los pensamientos, intereses, actividades, proyectos, planes y esperanzas de los otros están más o menos vinculados a la persona que muere y al hecho de su muerte". La naturaleza de este vínculo viene en parte por la pertenencia de las personas a una diversidad de estructuras sociales, como la familia, etc. Igualmente, "las muertes ocurren dentro de un orden médico organizacional"4.

Es evidente que todos vamos a morir aunque eso no quiere decir que aceptemos socialmente la muerte, suele ser vivida con rechazo y como algo que concierne a los otros. A la vez, cuando se personaliza, pertenece cada vez más a la esfera de lo privado y es difícil de tratar. La participación social, especialmente, en el entorno urbano está relegada a decisiones personales de cada grupo familiar y la función de acompañamiento y duelo que ha sido decisivo hasta el siglo XII, posteriormente fue modificándose progresivamente. En la actualidad, deja, cada vez más, de ser una ceremonia pública y organizada ${ }^{5}$.

El objetivo de este estudio ha sido conocer la opinión y actitudes de los pacientes, los familiares y el equipo interdisciplinar de atención hospitalaria, respecto a los cuidados paliativos y la relación que establecen los pacientes con su cuerpo, el dolor y la proximidad a la muerte.

\section{Discurso metodológico}

Este artículo forma parte de una investigación extensa sobre el proceso de morir de los pacientes con cáncer en situación terminal. Se realiza en un hospital oncológico dentro de la UCP. Tanto el hospital oncológico, como el hospital general de nivel III se encuentran dentro del Campus de Bellvitge de la Universidad de Barcelona. La investigación, resultado de una tesis doctoral, se realizó entre febrero del 1999 hasta diciembre de 
2001. Los escenarios en esta investigación incluyen la unidad de hospitalización (UH), la consulta externa (CE) y la unidad funcional interdisciplinar sociosanitaria (UFISS). La metodología utilizada es la cualitativa mediante la observación participante y la entrevista en profundidad, con soporte de entrevistas informales y material bibliográfico sobre el tema.

Así, la metodología cualitativa permite abordar la producción de significados que se construyen mediante las diferentes modalidades de interacción en el escenario de la paliación. Sus técnicas acercan a la investigadora al proceso de organización social de los enfermos en terminalidad, los familiares y los profesionales. Ayudan a construir los contextos, las necesidades y las acciones de los agentes que interactúan en cuidados paliativos. Todo ello para poder comprender el mundo propio de estos colectivos y la organización de la institución sanitaria presentada a través de las representaciones y las prácticas desarrolladas cotidianamente.

La observación se dirige al conjunto de los actores sociales de la UCP, enfermos, familiares y profesionales. En la observación se establecen unas estrategias de observación/participación que sirven de guía para adaptarse al funcionamiento del servicio y adentrarse en el trasfondo del mismo. La investigadora participa en charlas, pase de visitas y actividades propias de los cuidados, como curas, tratamientos, higienes, dietas, es decir, en todo lo relacionado con las actividades del enfermo, los familiares y los profesionales del servicio. Los pacientes son, en todo momento, informados del papel de la investigadora, y por un igual, los familiares. Los profesionales eran los que facilitaban, al inicio, el contacto con los enfermos y sus familiares. Además de la Unidad de Hospitalización (UH) la consulta externa y el hospital de día son otras áreas de observación y de acceso a los informantes.

La selección de los participantes procede del entorno del servicio de cuidados paliativos (SCP). El grupo de familiares son elegidos, en su mayor parte, entre los reconocidos como los cuidadores principales. En cuanto a los profesionales, se realiza la elección de los interlocutores que, por su trayectoria y formación, pueden aportar una mayor experiencia sobre la atención a los enfermos en estado terminal y a los familiares.

Se realiza las entrevistas en profundidad a once enfermos, entre los que figuran siete mujeres (entre 40 y 83 años) y cuatro varones (entre 52 y 58 años). La tipología de género no ha podido ser mantenida equitativamente porque el factor de vulnerabilidad de los informantes determina en muchas ocasiones la muestra. A 6 familiares, entre ellos hay 5 mujeres (entre 66 y 27 años) y un varón de 55 años. En esta tipología de género queda reflejada la característica de cuidadora en esta muestra. Y a 6 profesionales, mujeres entre $48 \mathrm{y}$ 24 años. Los profesionales entrevistados son una médica, dos enfermeras, una auxiliar de clínica, una trabajadora social y una psicóloga. Los informantes fueron elegidos por el tiempo que llevaban en la unidad, por la disciplina a la que pertenecían y por el grado de disponibilidad.

El orden que se constituye para las entrevistas es el de los pacientes, seguido de los familiares y posteriormente los profesionales. El enfermo es considerado, en todo momento, prioritario porque sus tiempos vienen determinados por el proceso de su enfermedad. En el caso de los familiares, se prioriza a los cuidadores principales de los enfermos previamente entrevistados, siempre que esto sea posible. Los profesionales son los últimos entrevistados, pues ayudan a cerrar el círculo informativo.

Las entrevistas a los pacientes están concertadas previamente y en coordinación con los profesionales responsables tanto de la unidad de hospitalización como de la consulta externa. El problema que se presenta es que, aunque los pacientes dan su consentimiento, en ocasiones, hay dificultades para realizarlas por alteraciones físicas y psicoemocionales inmediatas. En el caso de los familiares y profesionales las entrevistas se establecieron previo acuerdo y sin dificultades. Antes de realizar las entrevistas, la investigadora suele mantener más de una conversación y darse a conocer al posible interlocutor y a sus familiares.

La duración de las entrevistas es de 90’ para los pacientes y de 120' para los familiares y profesionales. Alguno de los pacientes llegó a dos horas. Todas las entrevistas fueron realizadas por la investigadora de este estudio y todas las sesiones fueron grabadas y a la vez se realizaban anotaciones específicas en el diario de campo. Una vez transcrita cada entrevista y revisada era consensuada con el informante correspondiente para aclarar dudas u omitir aquellos datos que no deseaba que quedaran reflejados. En esta investigación, se aplican las estructuras narrativas con el convencimiento de que se puede extraer el máximo de los relatos expuestos por los informantes.

El análisis de la información se lleva a cabo a partir la revisión de los diarios de campo y de las entrevistas grabadas. La información es agrupada en categorías en función de los objetivos marcados en la investigación. Los pasos realizados 
para ello son la creación de categorías, codificación y clasificación de las mismas ayudándose del programa Atlas.ti. Estas categorías aúnan las principales ideas extractadas de la OP y de las entrevistas en profundidad. Se reproducen literalmente, entre comillas, los testimonios de los informantes. En este artículo se presenta lo referente al cuidado paliativo y a la relación que establecen con el cuerpo y el dolor.

\section{Resultados del discurso etnográfico}

Los cuidados paliativos en la etapa final de la vida de un enfermo se acompañan, por lo general, de un gran impacto físico, emocional y social para el afectado y sus familiares, por lo que las metas básicas de la paliación son la búsqueda de su bienestar a través de un tratamiento adecuado del dolor y otros síntomas, el alivio y la prevención del sufrimiento con el mayor respeto a su dignidad y autonomía y el apoyo a su familia para que se enfrente mejor a la futura muerte y al duelo ${ }^{6-8}$.

Entre los testimonios presentados los profesionales dan su versión particular de los cuidados paliativos, como un espacio en el que el paciente es atendido en su proceso de enfermedad/ atención/muerte. Es el lugar en dónde proporcionan una atención holística a la persona en fase terminal y ofrecen un apoyo a los cuidadores principales (familiares). Así, los cuidados paliativos son concebidos desde la dimensión de cuidar a personas que necesitan ayuda tanto física como emocionalmente, es decir, holísticamente.

Por lo general los profesionales de CP suelen contar con una trayectoria profesional en la que han pasado por diversas unidades de diferentes hospitales y consideran que la acción de cuidar se puede desarrollar en toda su dimensión. Establecen una diferencia significativa, ya que consideran que la idea cambia, "Vamos a cuidar a personas, no a la enfermedad". La enfermedad es sustituida por la alteración de los síntomas que hay que tratar. Aunque en la atención a la persona en situación terminal tengan en cuenta las alteraciones de los síntomas y valoren por ejemplo el hematocrito, la albúmina, etc., estas acciones están en función de si se desestabiliza el estado general del paciente, si no, no son significativos los valores analíticos. El objetivo es conseguir que el paciente mantengan un estado general lo más confortable posible.

Para los enfermos y familiares la noción de $\mathrm{CP}$ está relacionada con su experiencia en el itinerario terapéutico, elaborado en el proceso de enfermedad/atención.
“SSabe usted qué es paliativos? Hombre, a mí nadie me ha explicado nada pero paliar... ¿No le han dicho los médicos? No, pero paliar, paliar, es una cosa que es aminorar efectos de lo que sea, o sea, no sé, cómo reducir daño o reducir sufrimiento, paliar es eso para mí, no sé ni lo he buscado en el diccionario pero pienso que eso, no lo sé usted me dirá". (Enferma Sra. Nena).

"¿Qué sabe usted de paliativos? ¿Qué es? Pues para mí... Yo qué sé, qué quieres que te diga. No te lo quiero ni decir... Porque paliativos es... pues mira, ir tirando. No es una cosa de curar ni nada, nada más que ir... Y eso, yo creo que él también lo sabe. Porque te da... bueno... Yo lo estoy viendo. Porque yo a veces veo enfermos aquí, que están en la consulta... y todos somos paliativos -todos-, aquí no hay ninguna persona que tenga unas anginas y viene y se visita y las anginas se le van a quitar; aquí no se va a quitar nada. Digo yo... ¿no será eso? ¿O qué crees tú? Dime tú lo que es". (Familiar Sra. Toñi).

Una gran mayoría, de los enfermos y sus familiares, conocen el significado y las implicaciones de estar ingresados y atendidos en cuidados paliativos.

Ratifico que los CP están sustentados en el cuidado y esta acción requiere de una formación por parte de todo el equipo interdisciplinar (médicos, enfermeros, trabajadores sociales, psicólogos, fisioterapeutas, etc.). Es notable que quienes cuentan con una trayectoria profesional y académica de cuidar son los profesionales enfermeros de CP. Así en su labor diaria son los que establecen las pautas de cuidado en el entorno de CP. El objetivo principal en $\mathrm{CP}$ es cuidar versus curar.

La acción de cuidar está relacionada directamente con el cuerpo, y en el quedan inscritos los cambios que experimenta en su proceso de vida. La enfermedad traza el camino hacia la terminalidad y la muerte. La persona en terminalidad percibe esas llamadas y es cuando suele cerciorarse de que está más próxima a su final.

El saber sobre el cuerpo, basado en la cultura y más o menos compartido por el conjunto de la comunidad, ha sido sustituido paulatinamente por un saber especializado. El saber biomédico es considerado la representación oficial del cuerpo humano de hoy, pero es un elemento de la cultura erudita sólo parcialmente compartido. La mayoría de las personas tiene un conocimiento bastante vago de su cuerpo. Por lo tanto, "en la conciencia de su fundamento físico, de la constitución secreta interna del cuerpo, el sujeto recurre paralelamente a muchas otras referencias" 9 . La relación con el cuerpo no se limita a una ima- 
gen del mismo, sino que también es una representación subjetiva constituida a partir de la representación objetiva del cuerpo producida y reenviada por los otros. Para Bourdieu, los esquemas de percepción y apreciación en los que el grupo sustenta sus estructuras fundamentales engendran las reacciones o las representaciones que el propio cuerpo suscita en los otros ${ }^{10}$.

Tras la experiencia de la enfermedad se presenta la problemática y la visibilidad del cuerpo $^{11}$. Los cambios de imagen trazan una línea divisoria entre el cuerpo sano y el portador de cáncer. En él quedan transcritos los efectos de la enfermedad y sus consecuencias. El cuerpo suele mostrarse desde dos perspectivas, una externa, que presenta la estructura física y las transformaciones que adopta, y otra interna, que transmite las sensaciones y emociones del individuo. El cáncer deja su huella en el cuerpo y cambia al individuo en lo físico. Con la enfermedad es el mundo lo que primero empieza a cambiar para el paciente. Estos cambios pueden ser interpretados como parte del proceso de la enfermedad, pero el cuerpo es a la vez objeto físico y sujeto de la experiencia y no pertenece a un mundo aparte. De esta manera, la enfermedad se encuentra presente en el mundo vital y es experimentada como un cambio en este último ${ }^{12}$.

El cambio de imagen en determinados tipos de cáncer es más visible e inquietante. Es el caso de un enfermo ingresado en el servicio en fase próxima a la muerte. Este enfermo, diagnosticado de neoplasia de laringe, tenía toda la zona de la mandíbula externa invadida (o desaparecida) por el cáncer, no se le entendía al hablar y su expresión era de gran angustia y nerviosismo. La mujer se encontraba a su lado con un semblante triste y resignado. Solían llamar con frecuencia al timbre y, al entrar en la habitación, aunque los profesionales y la observadora conocían el diagnóstico y el avance de la enfermedad, la expresión nos cambiaba sin poder apenas disimular. Aunque se colocaba con frecuencia una especie de barba postiza, esto hacía más grotesca la situación. Las dificultades de este paciente eran múltiples, se comunicaba con una libreta, no podía comer, era consciente de sus limitaciones y tenía que afrontar el rechazo controlado que ocasionaba su estado físico. La mujer permanecía triste y muy callada. Personalmente me creaba una sensación de impotencia y a la vez pienso que es uno de los peores cánceres que puede padecer un individuo. El principal factor influyente en el rechazo es la pérdida de imagen, ya que por mucho que los otros se preparen para normalizarlo es difícil lograrlo.
Estos enfermos crean un gran impacto en los profesionales de la unidad y sus familiares son transmisores de un gran sufrimiento.

El transcurso de la enfermedad exige a los afectados desarrollar una actitud adaptativa, lo que les permite mantener un cierto equilibrio ante unos acontecimientos que no pueden cambiar.

El deterioro de la imagen corporal se instaura de manera progresiva y continuada. En el cuerpo enfermo quedan transcritos los efectos de la enfermedad, lo que provoca intensas emociones como inseguridad, culpa y sufrimiento. Las percepciones que los pacientes tienen de su cuerpo suelen ser realistas, perciben el deterioro en todas las dimensiones que suele inquietarles. Paralelamente, entran en un proceso de adaptación/resignación que les permite en parte vivir con su enfermedad.

"Si yo me encontrara bien, lo mismo me daba. Más que estar guapa, estar bien. El estar más o menos, mira, a ver, dentro de no tener un buen tipo, tengo mejor tipo ahora porque estoy más delgada, que no que lo que yo soy, que yo soy de estar gordita (risas). Yo soy de estar llenita. Esta cara no es mi cara tampoco, estoy hinchada supongo que de la enfermedad y de la cortisona, de las dos cosas" (Enferma. Sra. Nena).

Otro hecho a señalar entre los que padecen cáncer, sobre todo en la fase terminal, es que suelen aparentar más edad de la que tienen en realidad. El enfermo percibe cómo progresivamente su cuerpo envejece cada día que pasa.

"Estoy más delgada que lo que yo soy y todo, parece el cuerpo parece de una persona, si yo tengo 55 pues dirías que es de una persona de sesenta y tantos años o sea la piel... mal como, como viejo, como todo, viejo, no sé cómo decirte. Sí, se cambia. No sé si es efecto de la enfermedad y de la quimio o sólo de la enfermedad o..., supongo que de todo junto". (Enferma. Sra. Nena).

Estos cambios físicos son los que más interrogantes, relacionados con su final, les plantean.

En cuanto a los familiares: "Y él.... viene... a lo mejor estamos sentados en un banco y viene... mira, el otro día sin ir más lejos (que la gente tampoco tiene cabeza), una persona mayor ya, pero una persona mayor, - ¿cuántos años tenía el hombre?... 92- (claro, Juan no está como estaba)... y le dijo que si era de su quinta. Y entonces él, como es una persona tan educada, le dijo: ya quisiera yo ser de su quinta y estar como está usted, porque ya tendría la vida vivida... ¿entiendes? Y otra persona le hubiera dicho... yo qué sé. Y él no se irrita por nada, ni le molesta que digas nada". (Familiar Sra. Toñi). 
La limitación de las capacidades físicas es una de las características principales de la enfermedad. La trayectoria de esta complica el curso diario de la vida del paciente, reduce sus fuerzas y le restringe en su vida cotidiana. Progresivamente cuentan con mayores impedimentos y son más frecuentes las limitaciones llegando a no poder ni desarrollar las actividades cotidianas. La agudización de los síntomas está presente en mayor o menor grado. La familia les sustituye en alguno de los roles e incluso suele limitarles actividades. Suelen ser muy conscientes de que en vez de mejorar cada vez se van deteriorando más.

"Los impedimentos físicos, es duro, no puedes hacer tu vida como la tienes que hacer. Lo de los huesos me limita, no soy capaz de coger un papel si cae al suelo. No sé cómo me voy a apañar cuando vaya a mi casa. Me preocupa ver que no sirvo pa nada. Que va a ser de mí, según estoy no me puedo valer, antes lo que no hacía yo, lo hacía mi marido, ¿y ahora?". (Enferma. Sra. Tico).

Los cambios corporales influyen en los cambios emocionales. En un primer momento el estado de ánimo suele estar alto, pero a medida que avanza la enfermedad suele decrecer.

"Cuando estoy mal, pues mira para estar... de la mejor manera que estoy es en la cama, en la cama y sin ganas de ver a nadie ni de hablar con nadie, ni..., y lo que sí que desde esta última recaída, tampoco tengo ganas de hablar..., que no tienes ganas de conversación tampoco. Mira, suerte de la tele porque la tele, te coge la cabeza y te la abstrae... lo único que me absorbe la cabeza y me quita de pensar y de eso pues es (la tele), que a veces no sé ni lo que veo tampoco, ¡eh! ...”. (Enferma. Sra. Nena).

Al deterioro corporal se suma el deterioro mental va aumentando de forma gradual dejando lagunas en el discurso, aunque sólo sea por momentos.

“¿Era verano? Sí, manga corta me parece que llevaba. Es que no me acuerdo. Sabes que pasa que con esta última quimio que me han dao. ¿Se le han olvidado cosas? Se me olvidan un montón de cosas. Hay cosas que me acuerdo y hay cosas que... Porque es que esta última sí que me ha dejao la cabeza...”. (Enferma. Sra. Nena).

Acostumbra a ser una constante que las personas afectadas se deterioren progresivamente física y psíquicamente-, pierdan su capacidad de autonomía y cada vez sean más dependientes del grupo familiar y profesional. Es una faceta en la que para algunos es mejor terminar, morir, y para otros a pesar de los obstáculos, seguir y aceptar o resignarse, posiblemente a la espera de que algo cambie su mala suerte. En esta situación lo sorprendente es que refieren una separación entre su cuerpo que no les responde y su mente que puede reproducir deseos y acciones inalcanzables en cada una de las etapas por las que pasan. La mayoría son conscientes que la etapa siguiente va a ser aún peor.

La enfermedad es la que modifica el cuerpo enfermo y la que le lleva a la pérdida de su identidad. El individuo no es el artífice de su modelación sino que es la enfermedad en su progresión la que inscribe en su cuerpo los cambios indefectibles en el proceso de enfermedad/atención/ muerte, hasta el final de su vida. Aunque consiga los mismos resultados o similares que puede llegar a desear o realizar un individuo, pero sin el consentimiento y deseo del afectado.

En cuanto a los testimonios del cuerpo y sus padecimientos, se parte de la idea de que "entender los padecimientos hace referencia a cómo los conjuntos sociales construyen, colectivamente, los procesos de salud/enfermedad por medio de un sistema de representaciones, entendidas como conjuntos de nociones, conocimientos, actitudes, imágenes y valores que se originan y comparten en sociedad"2.

Socialmente, la percepción que las personas tienen del cuerpo se debe, además de a una estructura física, a un conjunto de atributos sobre su significado social y psicológico y sobre su estructura y funcionamiento ${ }^{13}$. Para Bourdieu, la interpretación que nos hacemos del cuerpo como forma perceptible produce una impresión tanto en la conformación física del cuerpo como en la manera de presentarlo. Entre todas las expresiones de la persona es la que menos y la que más difícil se deja modificar en todas sus dimensiones. Para el autor es, por este hecho, interpretada socialmente como «la que expresa del modo más adecuado el ser profundo o la naturaleza de la persona al margen de toda intención significante ${ }^{10}$.

El sufrimiento se produce cuando el enfermo percibe un estímulo o su situación actual como una amenaza a su integridad biológica o psicológica, y se siente impotente, sin control ni recursos para afrontar dicha amenaza. La sensación de amenaza y el sentimiento de impotencia son subjetivos, por lo que el sufrimiento, por tanto, también lo será ${ }^{\text {. }}$

Al deterioro progresivo y limitante se le suma el dolor, a medida que avanza la enfermedad. Se concibe como una experiencia universal ya que el individuo lo padece y pasa en distintos momentos de su vida por diversas situaciones dolorosas. El dolor se percibe de manera individual, ya 
que no todas las personas lo sienten por igual, y esta percepción es el resultado de un aprendizaje sociocultural. Para David Le Breton, "el hombre es una consecuencia de las condiciones sociales y culturales, y también es un infatigable creador de significados con los cuales vive" 14 .

El dolor no es solamente un hecho biológico, sino que en este influyen aspectos emocionales a partir de los aprendizajes sociales y culturales adquiridos desde la infancia en el entorno familiar, por lo que no es un mero flujo sensorial, sino una percepción que en principio plantea la cuestión de la relación del mundo del individuo con la experiencia acumulada en relación con él. También el dolor lleva implícita tanto la sensación (sensory pain) como la percepción (suffering pain $)^{14}$.

La manifestación de dolor actúa como protección del organismo ante posibles peligros. Sin él, y sin la capacidad de sufrir, se comprometería la existencia humana debido a su vulnerabilidad. Su padecimiento prepara al individuo para identificarlo, fortalecerse y combatirlo cuando este se presenta.

El dolor es un hecho necesario para el ser humano, quien mantiene una relación directa con él, ya que "constituye uno de los elementos que conforman nuestra identidad como sujetos humanos y toda sociedad articula sistemas de cognición, comprensión y acción ante ese fenómeno" ${ }^{15}$ y resulta imprescindible para poder vivir. Pero el dolor no actúa siempre como protector de una enfermedad, sino que a veces, cuando se manifiesta, es demasiado tarde y apenas queda margen de actuación, como en el caso del cáncer. Es decir, "el dolor es una manifestación caprichosa que prosigue su camino torturando la existencia sin revelar nada apropiado para mejorar el estado del paciente" ${ }^{\prime 4}$.

Desde la perspectiva de la afectación del dolor y el desconocimiento de su intensidad y duración, es interesante el concepto de dolor total, establecido por Cicely Saunders, que lo enfoca no sólo como un dolor físico sino también social, emocional y espiritual. Es necesario tener en cuenta no solo la necesidad de analgésicos, sino además la comprensión humana y la ayuda social práctica. Así, "ningún paciente debería sufrir por culpa de un tratamiento inadecuado"16. Esta concepción del dolor total es el eje central de la filosofía de los hospices, formulada también desde los CP y aplicada a los pacientes en estos servicios.

A medida que avanza la enfermedad, la presencia del dolor suele ser más frecuente e intensa y pone a prueba la capacidad de control de los enfermos. El dolor es el punto central de la acción terapéutica en el entorno de cuidados paliativos. Las alusiones al dolor por parte de los sujetos son múltiples y diversas. Por lo general, el dolor se presenta como una experiencia insoportable y un anuncio de su propia muerte, para retornar a las esperanzas anteriores en cuanto este desaparece. Por lo que el cuerpo enfermo, con dolor, adquiere nuevos sentidos, interpela y es interrogado de forma renovada. El horizonte de finitud, la amenaza latente de la propia muerte como es el caso del cáncer, otorga a este fenómeno caracteres particulares ${ }^{11}$.

¿Cuando ha tenido dolor fuerte, ¿qué piensa? ¡Uf! Muy mal. Tirarme hasta por la ventana. Pero tirarme. Yo les decía: o me hacéis algo o me tiro por la ventana o hago alguna trastada. Porque era insoportable. Es que a mí ya no... me tenían que llevar a rastras. Mis pies no andaban; iba así como una inútil... Porque he sido un nervio. Y que te veas así... pues aún se te cae el mundo encima más... (Enferma. Sra. Alegre).

Por lo general, cuando pasa el dolor vuelven los deseos de vivir y luchar, sobre todo por la familia.

En cambio, para algunos pacientes el dolor no es significativo y cuando se manifiesta es esporádico y de fácil control, o es lo que perciben y transmiten.

No, no, no, no. Yo dolor... es que no tengo. Hombre, a veces tengo un poco dolor de barriga, aquello como retortijones que dicen, o parecido. Pero aquello de decir dolor, dolor, no. Este invierno me cogió un dolor aquí arriba en la espalda... Pero es que no tiene nada que ver, me parece, aquí arriba a la espalda con lo que tengo abajo en la barriga. ¿No? Tuve como un dolor que no me podía mover, dos o tres días. Algún día, pues me cogía dos veces. Me cogió un dolor, también hace más de un año, aquí al costado. Mira, me cogía la esterilla y me iba a la cama y me pasaba. Pero yo, quiero decir, la doctora del pueblo, que más o menos también me hace el seguimiento y eso, siempre me dice: cuando tengas dolor, tomate un Nolotil. (Enferma. Sra. Fina).

El dolor corporal, en el padecimiento de cáncer, puede ser el resultado del avance de la enfermedad. Se sitúa en el supuesto de enfermedad incurable, de la posible intolerancia al sufrimiento y de la percepción de proximidad, aunque sea por momentos, a la muerte. La relación con el dolor es de prioridad, por la necesidad de los enfermos de combatirlo o aliviarlo. Asimismo, cuando el dolor disminuye o desaparece los protagonistas reanudan su vida cotidiana. Según Le 
Breton, "en todo dolor hay en potencia una dimensión iniciática, un reclamo para vivir con mayor intensidad la conciencia de existir (...) el dolor padecido es antropológicamente un principio radical de metamorfosis, y de acceso a una identidad restablecida"14.

De la misma manera que en la enfermedad incurable el dolor y el sufrimiento marcan un itinerario y trazan unas huellas perceptibles en el cuerpo, estos signos pueden resultar una señal que alerta de la proximidad a la muerte. La mayoría de los pacientes manifiestan miedo a morir con dolor y a dejar a sus familiares, por lo que prefieren morir en el hospital más que en el domicilio. Aunque los avances científicos han influido positivamente en el control del dolor, en algunos casos, existen momentos críticos en los que se producen situaciones de descontrol y desesperación, de quien lo padece.

\section{Conclusiones}

La acción de cuidar, implica desarrollar acciones dirigidas a personas que necesitan seguimiento y control de los síntomas y una atención emocional. Por lo que la paliación implica la búsqueda de bienestar del paciente mediante un tratamiento adecuado ante el dolor y otros síntomas, así como el alivio y la prevención del sufrimiento respetando su dignidad y autonomía, y el apoyo a sus familiares para que puedan construir sus procesos de pérdida y duelo. En esa actuación queda implícito que cuidar es por un igual ayudar a morir como evitar la muerte, por lo que como individuos es algo que podemos necesitar, en un momento dado de nuestra vida, aunque en la actualidad esta atención no llega a toda la población.

Para los pacientes los cuidados paliativos son el lugar a donde van porque ya no pueden hacer nada por ellos, aunque manifiestan que nadie se lo ha dicho, son conscientes de que el itinerario recorrido en búsqueda de salud tiene su fin y están a la espera del desenlace final, con el deseo de no sufrir y ser cuidados hasta el final.

El cuerpo es el que exterioriza los cambios ocasionados por la enfermedad y da cuenta de que algo no va bien. Los enfermos son conscientes de que pierden sus capacidades y de que no hay vuelta atrás. La relación con el cuerpo aparece por momentos desligada de la realidad, como mecanismo compensatorio, para volver a cerciorarse de que los hechos están ocurriendo. Cuando el tratamiento no es efectivo son conscientes, aunque no lo manifiesten verbalmente, que el tiempo de curación da paso al de la paliación.

El sufrimiento físico y psicoemocional es una constante en CP que afecta tanto a quien lo padece como a los familiares y a los profesionales. A los enfermos que han padecido sufrimientos y dolores les inquieta que esos episodios se repitan, a los familiares que el enfermo pase por periodos de sufrimiento físico y psíquico como queda reflejado en las narraciones. En el caso de los profesionales de cuidados paliativos, tanto sufrimiento les cambia y afecta personalmente. La edad del paciente, si tiene hijos de edades tempranas, si han establecido lazos afectivos o la patología se manifiesta externamente, les afecta mucho más y manifiestan que no suelen tener un apoyo profesionalizado por la institución sanitaria, ya que en el espacio de la paliación es necesario para atender a los pacientes y familiares.

Durante la etapa de paliación, cuando se presenta el descontrol de los síntomas, entre ellos el dolor, es cuando se sienten más próximos a la muerte, al menos por momentos. Suelen manifestar que no tienen miedo a la muerte pero si a morir con dolor y dejar a sus familiares. En la fase final tienen un proceso de deterioro evidente que les puede concienciar de su propia muerte. 


\section{Referencias}

1. Bayés R. Afrontando la vida, esperando la muerte. Madrid: Alianza editorial; 2006.

2. Getino M. La espera. Construcción social de la muerte en el mundo de los cuidados paliativos. Barcelona: Laertes; 2012.

3. Sontag S. La enfermedad y sus metáforas. Madrid: Taurus; 1996.

4. Sudnow D. La organización social de la muerte. Buenos Aires (AR): Tiempo Contemporáneo; 1971.

5. Ariès Ph. La muerte en occidente. Barcelona: Argos Vergara; 1977.

6. Astudillo W, Mendinueta C. Guía de recursos sanitarios y sociales en la fase final de la vida en Guipúzcoa. San Sebastián: Sovpal; 2003.

7. Boceta J. Sobre cuidados paliativos (CP), atención en la agonía, sedación y otros asuntos. Medicina de familia 2005; 6(3):43-48.

8. Utor L. Capacitación de enfermería en cuidados paliativos. Medicina Paliativa 2007; 14(2):1-4.

9. Le Breton D. Antropología del cuerpo y modernidad. Buenos Aires: Nueva visión; 2002.

10. Bourdieu P. Notas provisionales sobre la percepción social del cuerpo. En: Mills W, Foucault M, Pollak M, Habermas N, Bourdieu P, Goffman E, Berstein B, Castell R. Materiales de sociología crítica. Madrid: La Piqueta; 1986. p. 183-194.
11. Alonso JP. Cuerpo, dolor e incertidumbre. Experiencias de la enfermedad y formas de interpelar el cuerpo en pacientes de cuidados paliativos. (Con)textos: revista d'antropologia $i$ investigació social 2008; (2):36-50.

12. Good B. Medicina, racionalidad y experiencia. Barcelona: Edic. Bellaterra; 2003.

13. Helman CG. Cultura, saúde e doença. Rio Grande do Sul, Brasil: Artes Médicas; 1994.

14. Le Breton D. Antropología del dolor. Barcelona: Seix Barral; 1999. p. 21;16;269-270.

15. Otegui R. Factores socioculturales del dolor y el sufrimiento. En: Perdiguero E, Comelles JM, editors. Medicina y cultura. Barcelona: Bellaterra; 2000.

16. Saunders C. Cuidados de la enfermedad maligna terminal. Barcelona: Salvat; 1980.

Artigo apresentado em 30/04/2013

Aprovado em 22/05/2013

Versão final apresentada em 22/05/2013 